\title{
Research on Dual-Wavelength Absolute Ranging Technology Based on FMCW Laser Interference
}

Bin Sun ( $D$ sun075634@163.com )

Xi'an Technological University

Junfang Song

Xizang Minzu University

Research Article

Keywords: FMCW, Dual-wavelength, Optical fiber, Laser interference, Absolute ranging

Posted Date: December 8th, 2021

DOI: https://doi.org/10.21203/rs.3.rs-1125684/v1

License: (c) (i) This work is licensed under a Creative Commons Attribution 4.0 International License. Read Full License 


\title{
Research on dual-wavelength absolute ranging technology based on FMCW laser interference
}

\author{
Bin Sun ${ }^{1}$, Junfang Song ${ }^{2}$ \\ 1.School of Optoelectronics Engineering, Xi' an Technological University, Xi' an, 710021, China \\ 2.School of Information Engineering, Xizang Minzu University, Xianyang 712082, China \\ (Corresponding author: Bin Sun; Email:sun075634@163.com)
}

\begin{abstract}
FMCW (Frequency-Modulated Continuous Wave) interferometer can achieve high-precision displacement measurement by phase discrimination of the interference signal. The phase needs to be superimposed continuously, so the optical path cannot be interrupted in the measurement process. To solve this problem, a new absolute ranging technology - decimal comparison method is proposed in the manuscript. According to the principle of FMCW interference, two DFB lasers with different central wavelengths are used to measure the same target. The sampled interference signal is processed by digital signal processing to calculate the integer and decimal of the synthetic wavelength period. The optical path difference of the target is calculated by the established mathematical model. The experimental results show that this method not only ensures the measurement accuracy, but also realizes the absolute measurement, and expands the application range of FMCW interferometry.
\end{abstract}

Key words: FMCW, Dual-wavelength, Optical fiber, Laser interference, Absolute ranging

\section{Introduction}

Laser has the characteristics of monochromaticity, coherence and parallelism, and plays an important role in the field of distance measurement [1-3]. At present, the commonly used laser ranging technology is mainly divided into two categories: incoherent measurement and coherent measurement. Among them, non-coherent measurement mainly includes pulse method and phase method. Coherent measurement mainly includes femtosecond optical comb method, multi-wavelength interferometry and frequency modulated continuous wave interferometry. The precision displacement measurement technology represented by the laser interferometer has been extremely widely used in the field of ultra-precision machining and detection [4-5].

The traditional laser heterodyne interferometer uses two kinds of monochromatic light with different frequencies, which is mixed by the photodetector and outputs the difference frequency signal. After demodulation, the optical path difference with large range and high precision can be obtained. Because it can only measure the relative displacement, in the measurement process, the target must move along the fixed guide rail and the optical path must not be interrupted, so it cannot meet the absolute measurement requirements of high-precision dimension detection. In order to solve this problem, laser interference absolute distance measurement technology has been studied by more and more scholars. In the 1970s, J. C. Wyant and C. Polhemus in the United States carried out dual wavelength interference experiments [6-8], and proposed that multiple wavelengths be combined to form synthetic wavelength chains with different lengths. At the same time, the measured distance is refined step by step from different synthetic wavelengths, which has become the basic theory of multi wavelength ranging. Since then, the research on multi 
wavelength ranging has developed rapidly [9-11]. In 2006, Nicolas Schuler of Germany and Yves Salvade of Switzerland proposed the multi wavelength ranging method of reference optical frequency comb [12]. In 2010, Liang Jing and others in China used a 633nm dual longitudinal mode HeNe laser and a $629 \mathrm{~nm}$ HeNe laser to form a composite wavelength of $117 \mu \mathrm{m}$, which further improved the measurement accuracy [13]. The above multi-wavelength ranging method comes from single frequency laser interferometry. The measurement system is complex and has poor stability and accuracy.

FMCW laser interferometry originated from radio frequency modulated continuous wave radar [14-15]. The basic principle is to implement periodic continuous frequency modulation on the single frequency laser, so that the modulated laser can be divided and recombined in the interferometer. When the optical paths of the reference arm and the signal arm of the interferometer are different, there is a certain frequency difference between the two FMCW lasers after beam combination, resulting in a dynamic beat interference signal. By measuring the change of the initial phase of the interference signal, the change of the optical path difference can be calculated. FMCW laser interference technology produces a dynamic mixing interference signal. It is easy to subdivide the phase, distinguish the phase moving direction and count the whole period, so FMCW laser interferometry can realize high-precision displacement measurement [16-17]. Based on the relative measurement of the FMCW laser interferometer, the manuscript proposes a dual-wavelength absolute distance measurement method - the decimal contrast method. It solves the problem that traditional interferometry is relative measurement and the optical path cannot be interrupted, thereby expanding the application of FMCW interferometry technology.

\section{FMCW ranging principle}

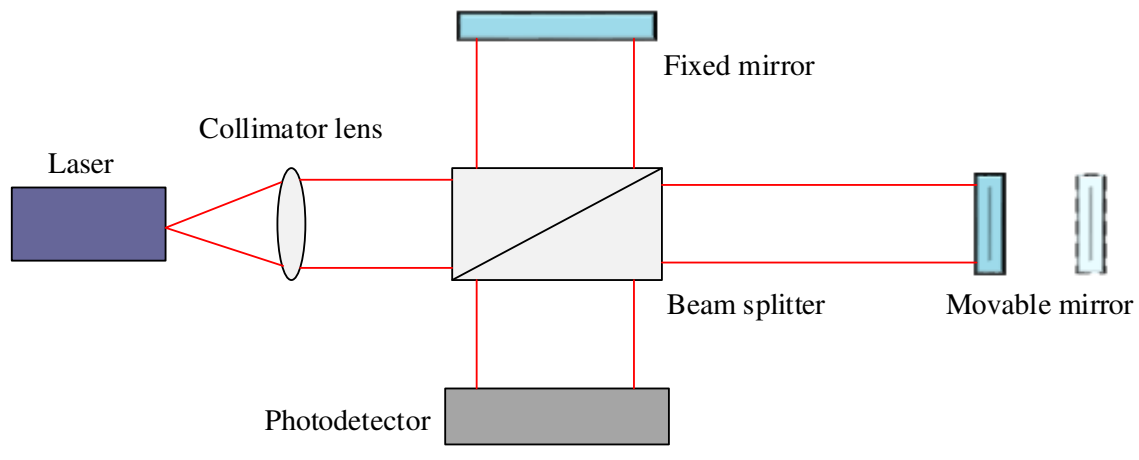

Fig.1 FMCW optical system diagram

As shown in Fig.1, the FMCW laser is collimated by a collimating lens and then divided by a beam splitter. The optical path where the fixed reflector is located is the reference beam, and the OPD will not change. The optical path where the movable reflector is located is a signal beam. When the reflected beam from the movable reflector and the fixed reflector meet at the beam splitter, the two beams will interfere to generate a dynamic interference signal. The displacement of the movable reflector is calculated by the frequency and phase of the interference signal.

When these two waves interfere with the light intensity of the signal, the OPD between the reference wave and the signal wave in one modulation period is as follows:

$$
\begin{aligned}
I(O P D, t) & =I_{0}\left[1+V \cos \left(\frac{2 \pi \Delta v v_{m} O P D}{c} t+\frac{2 \pi}{\lambda_{0}} O P D\right)\right] \\
& =I_{0}\left[1+V \cos \left(2 \pi v_{b} t+\phi_{b 0}\right)\right]
\end{aligned}
$$


Where $\Delta v$ is the optical frequency modulation excursion, $v_{m}$ is the frequency the modulation signal, $c$ is the speed of light in free pace, $\lambda_{0}$ is the central optical wavelength, $v_{b}$ and $\phi_{b 0}$ are the frequency and the initial phase of the beat signal.

$$
\phi_{b 0}=\frac{2 \pi O P D}{\lambda_{0}}
$$

It can be seen from equation (6) that each time the OPD changes by one wavelength, the initial phase of the cosine signal changes $2 \pi$, and the beat signal shifts by one cycle. Therefore, the OPD can be calculated by measuring the amount of change (phase shift) of the initial phase of the beat signal. Usually, the calculation of $\phi_{b 0}$ uses the cycle counting method, which continuously records and accumulates the amount of change in the phase shift during the measurement to calculate the total phase shift amount $\Delta \phi_{b 0}$ during the entire measurement process.

\section{Dual-wavelength absolute ranging method based on FMCW}

According to the principle of FMCW laser interferometry, two independent interference light paths are designed to measure the same measured target with laser light sources with different central wavelengths, as shown in Figure 2.

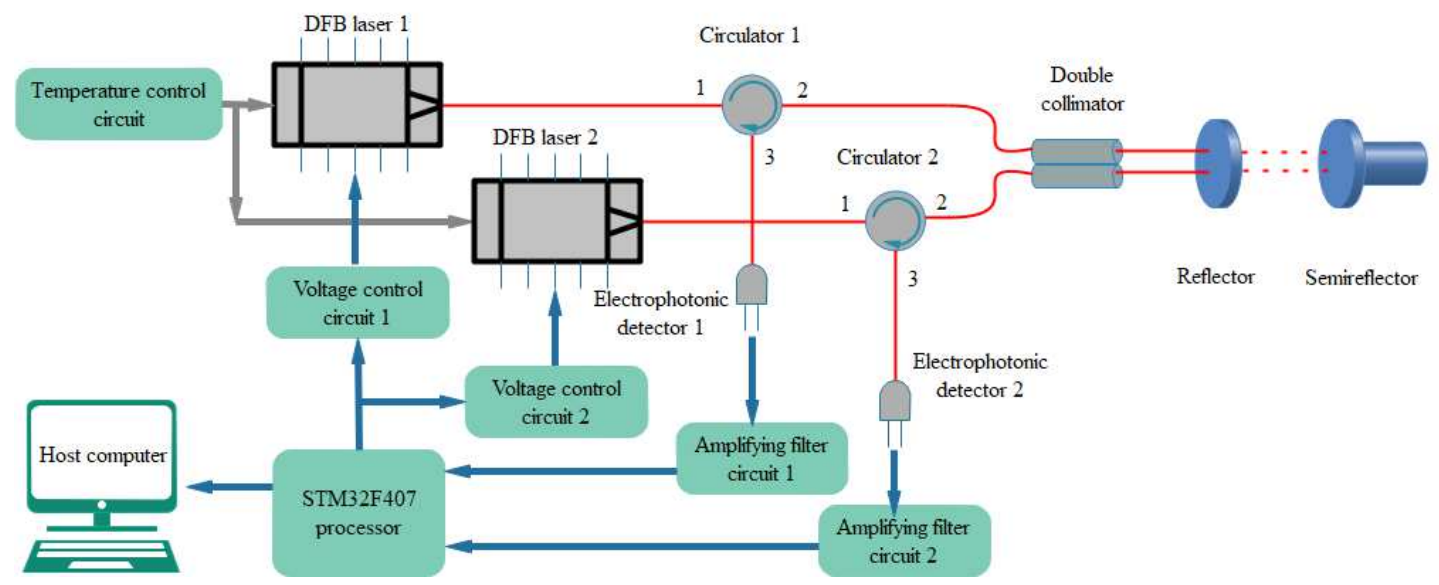

Fig.2 Schematic diagram of dual light source optical path

The two laser light sources emit light beams with wavelengths of $\lambda_{1}$ and $\lambda_{2}$, respectively, which form interference after passing through the Michelson interferometer, and the mixing signals are obtained by the two detectors respectively.According to formula (2), there are the following formulas:

$$
\begin{aligned}
& O P D=\left(N_{1}+\varepsilon_{1}\right) \lambda_{1} \\
& O P D=\left(N_{2}+\varepsilon_{2}\right) \lambda_{2}
\end{aligned}
$$

Where $N$ is the integer of the interference signal period, and $\varepsilon$ is the decimal of the interference signal period.

Since two laser waves measure the same F-P cavity at the same time, the optical path difference OPD measured by the two laser waves is equal. From the formula and (3) and (4), it can be 
concluded that:

$$
N_{1} \lambda_{1}+\varepsilon \lambda \overline{\overline{1}} N \lambda_{2}+\varepsilon \lambda
$$

When the wavelengths of the two lasers $\lambda_{1}$ and $\lambda_{2}$ are similar, there are:

$$
N_{1}=N_{2}=\frac{\varepsilon_{2} \lambda_{2}-\varepsilon_{1} \lambda_{1}}{\lambda_{1}-\lambda_{2}}
$$

As can be seen from formulas (3) and (6), two columns of mixed signals are superimposed to form a synthetic signal. Through the small value of the two interference wave periods, the integer of the number of synthetic signal periods can be calculated, and then the OPD can be calculated accurately. Compared with the original method that needs to continuously stack and count the phase of the mixed signal, this technology only needs to calculate the initial phase of the two measurement signals. Therefore, even if the optical path in the measurement is interrupted, the absolute measurement of OPD can be realized by this technology.

It can be seen from the above formula that the measured distance is determined by the synthetic wavelength, which is equivalent to interferometric ranging with a laser beam with a wavelength equal to the synthetic wavelength. If the wavelengths of the two selected laser sources are relatively close, the synthetic wave is longer than any wavelength. When the wavelength is reasonably selected, the measured distance can be obtained by calculating the fractional part of the phase of the mixing signal. This technology can also be extended to multi wavelength measurement of target distance. When the measured distance is large, multiple laser sources can be selected for synthetic measurement. Multiple synthetic wavelengths form a synthetic wavelength chain to gradually approach the measurement results, so as to improve the measurement accuracy.

\section{Experimental verification}

As can be seen from Fig.2, the measurement system uses two independent laser light sources to measure the same target mirror after passing through two parallel collimators through linear frequency modulation. The reference light and the measurement light form an interference signal, which is collected by two independent photodetectors. Finally, the microprocessor stm32f 407 demodulates and calculates the OPD information.

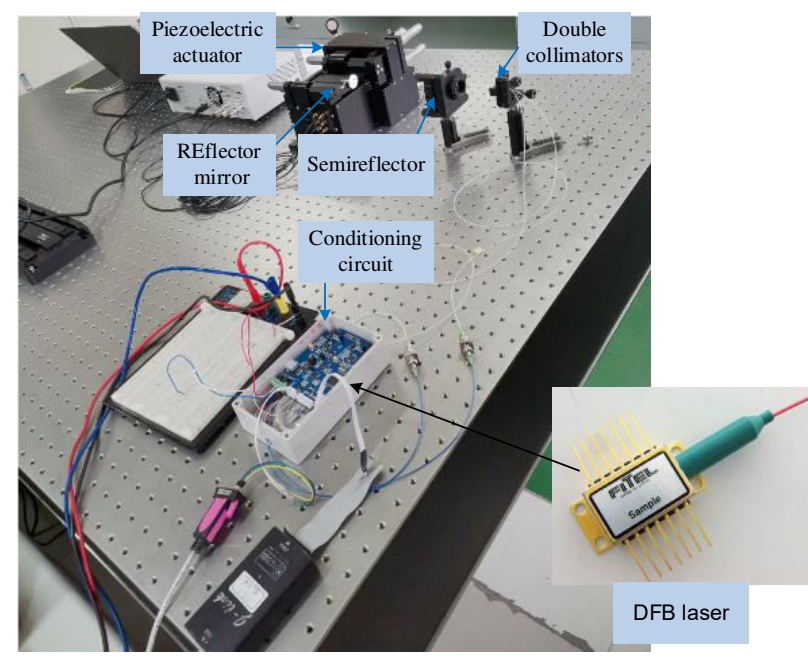

Fig.3 Measurement experiment 
Fig. 3 is the actual diagram of the FMCW laser interference dual-wavelength absolute ranging experiment. The experimental equipment includes Optical platform, Double collimator (two collimators are absolutely parallel), Semi-reflector, Reflector, laser, Piezoelectric actuator and Control circuit. Among them, the Piezoelectric actuator has a maximum stroke of 18 microns, a linearity of $0.1 \%$ F.S, a repeat positioning accuracy of $0.05 \%$ F.S, and a displacement resolution of $0.1 \mathrm{~nm}$.

After the frequency of the DFB laser is linearly modulated, two absolutely parallel collimators emit modulated light beams. The reference light returns through the semi-transmissive reflector, and the measurement light returns from the opaque target reflector after passing through the semi-transmissive reflector. The reference light and the measuring light are combined in the photodetector to generate a mixing signal. The control circuit collects the mixing signal in the photodetector, calculates the fractional part and the number of whole cycles through digital signal processing, and then calculates the displacement information of the target reflector by formula (6). The target reflector is placed on the driving piezoelectric actuator. Adjust the linear change of optical path difference that drives the piezoelectric actuator to move back and forth. The displacement trajectory demodulated from the mixing signal is compared with the known linear trajectory of the piezoelectric actuator in order to verify the correctness of the FMCW laser interference dual-wavelength absolute ranging method.

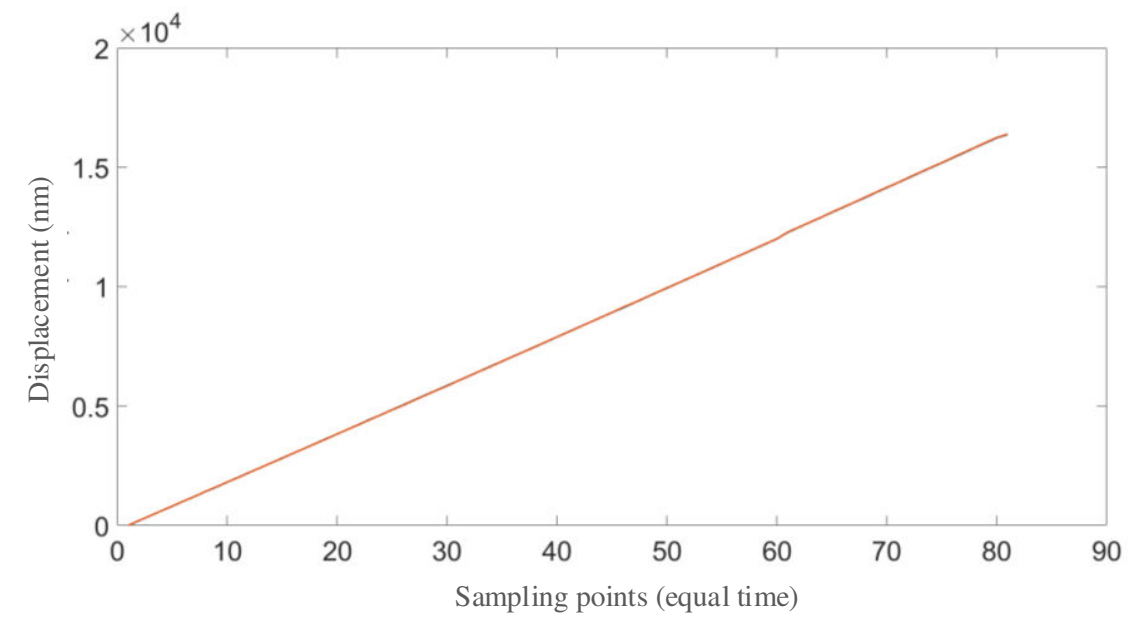

Fig. 4 Linear variation curve of displacement measured in experiment

During the experiment, the FMCW laser interferometer continuously measures the movement of the target reflector and draws the trajectory curve of the optical path difference. The laser in the experiment was produced in FRL15DCWx series of DFB laser module from FITEL, Japan. The wavelength of this laser has been rigorously calibrated before it is sold. The center wavelengths are $1550.12 \mathrm{~nm}$ and $1580.35 \mathrm{~nm}$, and the maximum absolute error of the interference level decimal is \pm 0.0048 . The experimental result is shown in Fig.4, the displacement measured by the 
interferometer is $15800 \mathrm{~nm}$. Compared with the known linear trajectory of the piezoelectric actuator, the displacement measured by the interferometer has a maximum linear error of $\pm 7.4 \mathrm{~nm}$. The FMCW laser interferometer continuously collects 17,000 data in a static state. A part of the scatter plot is shown in Fig.5. The scatter diagram is processed with normal distribution. Within the $95 \%$ confidence interval, the maximum standard deviation is $\pm 15 \mathrm{~nm}$. The scatter diagram shows the stability of FMCW laser interferometer.

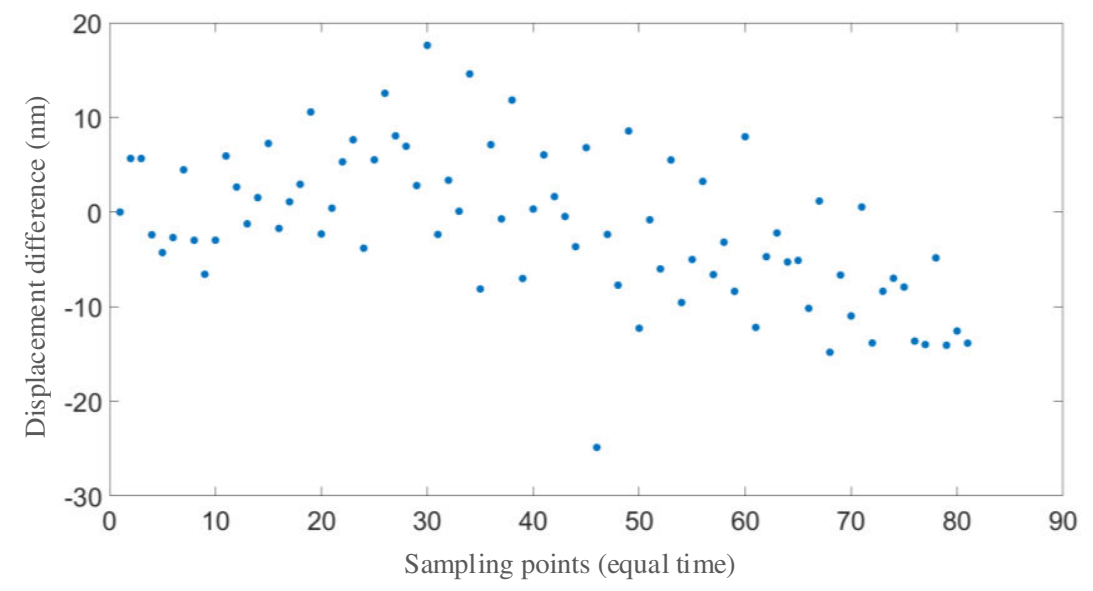

Fig.5 Scatter diagram of displacement error measured in experiment

\section{Conclusion}

FMCW laser interferometer usually takes phase as parameter to realize high-precision displacement measurement. Because the phase of the mixed signal needs to be superimposed continuously in the measurement, the optical path cannot be interrupted in the measurement process, otherwise the loss of phase information will lead to an error in the calculation of displacement information. To solve this problem, a new absolute ranging technology - decimal comparison method is proposed in the manuscript. Through theoretical analysis, the calculation model of optical path difference of fractional comparison method in synthetic wavelength is established. Then, the effectiveness of the method is verified by accuracy experiments. The experimental results show that the maximum allowable error of displacement measurement is $\underline{ \pm}$ $15 \mathrm{~nm}$, and the decimal comparison method can have high accuracy and stability.

\section{Author contribution statement}

Author Bin Sun completed the experimental design and the main framework of the manuscript, author Junfang Song completed data acquisition and algorithm verification, and polished the language of the manuscript.

\section{Funding}

Supported by National Natural Science Foundation of China (No.62041305), Xizang Natural Science Funds (No.XZ202001ZR0065G) and Shaanxi Natural Science Funds (No. 2021JM-437/2021JM-438).

\section{The data availability statement}

The data used to support the findings of this study are available from the corresponding author 
upon request.

\section{Disclosures}

The authors declare no conflicts of interest.

\section{Reference}

[1] Wu H, Zhang F, Cao S, et al. Absolute distance measurement by intensity detection using a mode-locked femtosecond pulse laser[J]. Optics Express, 2014, 22(9):10380-97.

[2] Jin, Jonghan, Kim, YoungJin, Kim, Yunseok. Absolute distance measurements using the optical comb of a femtosecond pulse laser[J]. International Journal of Precision Engineering \& Manufacturing, 2010, 8(4):22-26.

[3] Wu H, Zhang F, Li J, et al. Intensity evaluation using a femtosecond pulse laser for absolute distance measurement[J]. Applied Optics, 2015, 54(17):5581.

[4] Guodong L, Cheng L, Bingguo L, et al. Combining sub-Nyquist sampling and chirp decomposition for a high-precision and speed absolute distance measurement method[J]. Applied Optics, 2016, 55(35):9974-9977.

[5] Lu C, Liu G, Liu B, et al. Absolute distance measurement system with micron-grade measurement uncertainty and $24 \mathrm{~m}$ range using frequency scanning interferometry with compensation of environmental vibration[J]. Optics Express, 2016., 24(26): 30215-30224.

[6] Polhemus C, Chocol C. Method for subtracting phase errors in an interferometer[J]. Applied Optics, 1971,10(2):441.

[7] Wyant J C. Testing Aspherics Using Two-Wavelength Holography[J]. Applied Optics, 1971,10(9):2113-2118.

[8] Polhemus C. Two-Wavelength Interferometry[J]. Applied Optics, 1973,12(9):2071-2074.

[9] Daendliker R , Hug K , Politch J , et al. High-accuracy distance measurement with multiple-wavelength interferometry[J]. Optical Engineering, 1995, 34(8):2407-2412.

[10] Yin, Chunyong. Absolute length measurement using changeable synthetic wavelength chain[J]. Optical Engineering, 2002, 41(4):746-750.

[11] Suzuki T , Matsuda M , Sasaki O , et al. Laser-diode interferometer with a photothermal modulation[J]. Applied Optics, 1999, 38(34):7069.

[12] Schuhler N, Yves Salvadé, Samuel Lévêque, et al. Frequency-comb-referenced two-wavelength source for absolute distance measurement[J]. Optics Letters, 2006, 31(21):3101-3.

[13] Liang Jing, long Xingwu, Zhang Bin, et al. Absolute distance interferometry using $117 \mu \mathrm{m}$ synthetic wavelength [J]. Acta optica Sinica, 2010, 30 (010): 2947-2951.

[14] Zheng J. Reflectometric fiber optic frequency-modulated continuous-wave interferometric displacement sensor[J]. Optical Engineering, 2005, 44(12): 124404.

[15] Zheng J. Optical frequency-modulated continuous-wave interferometers[J]. Applied Optics, 2006, 45(12): 2723-2730.

[16] Zheng J. Reflectometric fiber optic frequency modulated continuous wave interferometric displacement sensor[J]. Optical Engineering, 2005, 44(12), 124401-124405.

[17] Zheng, Jesse. Single-Mode Birefringent Fiber Frequency-Modulated Continuous- Wave Interferometric Strain Sensor[J]. IEEE Sensors Journal, 2009, 10(2):281-285. 